\title{
THE EVOLUTION OF DUST EMITTED BY A UNIFORM SOURCE ABOVE GROUND LEVEL
}

\author{
I. A. ELTAYEB and M. H. A. HASSAN
}

Received 18 June 2001 and in revised form 21 January 2002

\begin{abstract}
A uniform source situated at a fixed location starts to emit dust at a certain time, $t=0$, and maintains the same action for $t>0$. The subsequent spread of the dust into space is governed by an initial boundary value problem of the atmospheric diffusion equation. The equation has been solved when the wind speed is uniform and diffusion is present both along the vertical and the horizontal for a general source. The solution is obtained in a closed form. The behaviour of the solution is illustrated by means of two examples, one of which is relevant to industrial pollution and the other to the environment. The solution is represented in graphic form. It is found that the spread of dust into space depends mainly on the type of source and on the horizontal component of diffusion. For weak diffusion, the dust travels horizontally with a vertical front at the uniform speed of the flow. In the presence of horizontal diffusion, dust diffuses vertically and horizontally. For a point source, the distribution of dust possesses a line of extensive pollution. For a finite-line source, the dust concentration possesses a point of accumulation that moves both horizontally and vertically with time.
\end{abstract}

2000 Mathematics Subject Classification: 35K15, 35Q35, 49K20, 35C15.

1. Introduction. The transport of small solid particles on the surface of the earth and in its vicinity is relevant to a wide spectrum of applications (see, e.g., [4]) which range from soil erosion $[7,16]$ to water pollution and sedimentation [10], to snow drift [14, 17], and to environmental pollution [6, 9, 15]. In arid and semiarid lands, such situations occur in deserts and areas close to deserts and the spread of these particles can cause considerable damage to agricultural lands, roads, and health. The particles span a wide range in size (diameters varying from less than $0.1 \mathrm{~mm}$ to $0.5 \mathrm{~mm}$ ) and the dynamics of the particles depends on their size. For particles of diameter $0.1-0.5 \mathrm{~mm}$, the particles remain on the surface or very close to it due to the strong influence of gravity and can lead to the formation of sand dunes [5]. For particles with diameters less than $0.1 \mathrm{~mm}$, the influence of gravity is much smaller and the particles form a suspension as they are transported by wind. It is this type of particles that is relevant to soil erosion and environmental pollution, and is also of great relevance to industrial pollution, as for example in the case of fumes emitted by industrial chimneys. The present study is concerned with such particles. 
The transport of such dust particles is usually governed by the atmospheric diffusion equation

$$
\frac{\partial c^{*}}{\partial t^{*}}+\mathbf{u} \cdot \nabla c^{*}=\nabla \cdot\left(\mathbf{D} \cdot \nabla c^{*}\right)-\mathbf{w} \cdot \nabla c^{*}, \quad \nabla \cdot \mathbf{u}=0
$$

in which $\mathbf{u}$ is the wind velocity, $\mathbf{w}$ is the settling velocity, and $\mathbf{D}$ is the stress tensor. The settling velocity depends on the particle size and can be taken as a measure of the influence of gravity. The stress tensor is a function of position and its dependence in different directions may be different so that it is not isotropic. The velocity, in general, depends on the position as well as time. The second term on the left-hand side of the first equation in (1.1) represents the advection of the particles by the local fluid motion.

The atmospheric diffusion equation has been solved in a variety of situations (see, e.g., [2, 11, 12, 13]). In all these cases, the stress tensor and velocity were assumed to take reasonably simple forms, with the most complicated forms being when one or both varied as a power law of the vertical coordinate. The reason for this is that complicated forms pose equations which cannot be solved analytically. Since the domain of influence of velocity on dust particles of interest is quite small, in terms of atmospheric dimensions, these simple forms are adequate since they can be considered as first terms in Taylor series expansions for these functions.

We will assume that the stress tensor has the form

$$
\mathbf{D}=\left(\begin{array}{ccc}
\gamma & 0 & 0 \\
0 & 0 & 0 \\
0 & 0 & \lambda z^{*}
\end{array}\right)
$$

in which $\gamma$ and $\lambda$ are constants, and the flow $\mathbf{u}$ is unidirectional and uniform so that

$$
\mathbf{u}=(U, 0,0), \quad \mathbf{w}=(0,0, W)
$$

in a Cartesian system of coordinates in which the $z$-axis is directed vertically upwards and the $x$-axis along the horizontal wind speed.

Most previous studies have concentrated on the steady-state solution of the diffusion equation (1.1). One of our interests here is to examine how such a steady state can be achieved. This requires the consideration of an initial boundary value problem in which case the time derivative is fully potent so that the equation is predictive. This allows us to examine the dependence of the solution on the initial conditions with a view to identifying the manner in which the steady-state solution is achieved. For this reason, we will consider a situation for which the steady-state solution is known [6].

In Section 2, we define the initial value problem and boundary conditions. In Section 3, we obtain the general solution in closed form using both Weber and Laplace transforms. Here, we examine certain known limiting cases and obtain 
simplified solutions for them. We also examine the case when time increases indefinitely and obtain a limiting solution which matches the already known solution of the steady-state problem. In Section 4, we examine the solution in detail and illustrate its dependence on the parameters of the problem as it evolves with time. Section 5 is devoted to a few concluding remarks.

2. Formulation of the problem. Consider a source at $x=0$ emitting dust at a prescribed (measured) rate. Such a situation may arise when dust passing through a certain position is measured at various levels. We intend to examine the development of the distribution of dust with time until a steady state is reached. Define a Cartesian system of coordinates $O\left(x^{*}, y^{*}, z^{*}\right)$ in which $O z^{*}$ is vertically upwards and $O x^{*}$ and $O y^{*}$ are horizontal. The concentration $c\left(x^{*}, z^{*}, t^{*}\right)$ of pollutant particles is governed by (1.1), (1.2), and (1.3). We assumed that the concentration is independent of the lateral coordinate $y^{*}$, and depends on the time $t^{*}$. Thus (1.1) takes the form

$$
\frac{\partial c^{*}}{\partial t}+U \frac{\partial c^{*}}{\partial x^{*}}=\frac{\partial}{\partial x^{*}} \gamma \frac{\partial c^{*}}{\partial x^{*}}+\frac{\partial}{\partial z^{*}} \lambda z^{\partial c^{*}} \partial W \frac{\partial c^{*}}{\partial z^{*}}
$$

where $U, W, \gamma$, and $\lambda$ are defined in (1.2) and (1.3).

If we define

$$
b=\frac{\gamma}{\lambda}\left(\frac{\lambda}{U}\right)^{2}, \quad v=\frac{W}{2 \lambda}, \quad x=\left(\frac{\lambda}{U}\right) x^{*}, \quad t^{*}=\frac{t}{\lambda}, \quad z=z^{*},
$$

we can write (2.1) in the neat form

$$
\frac{\partial c^{*}}{\partial t}+\frac{\partial c^{*}}{\partial x}=(2 v+1) z \frac{\partial c^{*}}{\partial z}+\frac{\partial^{2} c^{*}}{\partial z^{2}}+b \frac{\partial^{2} c^{*}}{\partial x^{2}}
$$

This equation is solved subject to boundary and initial conditions. Since the source is switched on at a certain time, we will assume that the concentration vanishes everywhere for $t \leq 0$. The distribution of the concentration can be assumed to satisfy one or more conditions at the ground level. We will assume here that the concentration vanishes at the top of the roughness layer, $z=z_{0}$. Another possible condition is the vanishing of the flux, which demands that $\partial c^{*} / \partial z=0$ at $z=z_{0}$. However, the imposition of the latter boundary condition will render the inversion of the transforms encountered in the solution more difficult. Since the subsequent distribution of the dust is entirely due to the emission by the source at $x=0$, we can impose the condition that it decays to zero faraway from $x=0$. The initial condition can be written in the form

$$
c^{*}(0, z, t)=Q f(z) H(t)
$$


in which the Heaviside function is defined by

$$
H(x-a)= \begin{cases}1 & \text { for } x>a \\ 0 & \text { for } x<a\end{cases}
$$

and $f(z)$ is an arbitrary function representing the variation of the source with height while $Q$ measures the amplitude of the source.

If we define

$$
q(x, \varsigma, t)=\varsigma^{\nu} c(x, \varsigma, t), \quad \varsigma=2 z^{1 / 2}, c=Q c^{*},
$$

we can rewrite (2.3) as

$$
\frac{\partial q}{\partial t}+\frac{\partial q}{\partial x}=\frac{1}{\varsigma} \frac{\partial}{\partial \varsigma}\left(\varsigma \frac{\partial q}{\partial \varsigma}\right)-\frac{\nu^{2}}{\varsigma^{2}} q
$$

If we make the further transformation

$$
u(x, \varsigma, t)=e^{-x / 2 b} q(x, \varsigma, t),
$$

we obtain

$$
\frac{\partial u}{\partial t}=\frac{1}{\varsigma} \frac{\partial}{\partial \varsigma}\left(\varsigma \frac{\partial u}{\partial \varsigma}\right)-\frac{v^{2}}{\varsigma^{2}} u+b \frac{\partial^{2} u}{\partial x^{2}}-\frac{1}{4 b} u
$$

The boundary and initial conditions can also be transformed to $u$ :

$$
\begin{gathered}
u\left(x, \varsigma_{0}, t\right)=0, \\
c(x, \varsigma, t) \longrightarrow 0 \quad \text { as } \varsigma \rightarrow \infty, \\
u(0, \varsigma, t)=H(t) f\left(\frac{\varsigma^{2}}{4}\right), \\
u(x, \varsigma, 0)=0 \quad \forall x, \varsigma \geq 0 .
\end{gathered}
$$

We are therefore required to solve (2.9) subject to the initial boundary conditions (2.10).

3. The solution. The solution is obtained by adopting the Weber transform defined by

$$
\tilde{q}(x, y, p)=\int_{z_{o}}^{\infty} q(x, y, z) H_{v}(p z) z d z
$$

in which

$$
H_{v}(p z)=J_{v}(p z) Y_{v}\left(p z_{o}\right)-J_{v}\left(p z_{o}\right) Y_{v}(p z)
$$

together with its inverse transform

$$
q(x, y, z)=\int_{0}^{\infty} \frac{\tilde{q}(x, y, p) H_{v}(p z)}{J_{v}^{2}\left(p z_{o}\right)+Y_{v}^{2}\left(p z_{o}\right)} p d p .
$$


In (3.2), $J_{v}(x)$ and $Y_{v}(x)$ are Bessel functions of the first and second kinds with order $v$ and argument $x$.

If we apply the transform (3.1) to (2.9), we get

$$
\frac{\partial \bar{u}}{\partial t}=b \frac{\partial^{2} \bar{u}}{\partial x^{2}}-\left(p^{2}+\frac{1}{4 b}\right) \bar{u}
$$

By taking the Laplace transform of (3.4) in time and using (2.10d), we find that

$$
s \hat{\bar{u}}(x, p, s)=b \frac{\partial^{2} \hat{\bar{u}}}{\partial x^{2}}-\left(p^{2}+\frac{1}{4 b}\right) \hat{\bar{u}} .
$$

If we apply both transforms to (2.10c) in the same order, we obtain

$$
\hat{\bar{u}}(0, p, s)=F(p) \frac{1}{s}
$$

in which

$$
F(p)=\int_{\varsigma_{0}}^{\infty} f\left(\frac{\varsigma^{2}}{4}\right) H_{v}(p \varsigma) \varsigma^{v+1} d \varsigma
$$

Equation (3.5) can be solved together with (2.10d) and (3.6) to find that

$$
\hat{\bar{u}}(x, p, s)=F(p) \frac{1}{s} \exp \left\{-a \sqrt{s+c^{2}}\right\}, \quad a=\frac{x}{\sqrt{b}}, c=\sqrt{p^{2}+\frac{1}{4 b}} .
$$

The next step is to invert the two transforms. Inverting the Laplace transform in $t$ gives

$$
\bar{u}(x, p, t)=F(p) G(x, t ; p),
$$

where $G(x, t ; p)$ is obtained by the use of a combination of shift and convolution theorems. Thus

$$
\begin{aligned}
G(x, p, t) & =\frac{x}{2 \sqrt{\pi b}} \int_{0}^{t} \tau^{-3 / 2} \exp \left(-c^{2} \tau-\frac{x^{2}}{4 b \tau}\right) d \tau \\
& =\frac{x}{2 \sqrt{\pi b t}} \int_{1}^{\infty} \frac{1}{\sqrt{v}} \exp \left(\frac{c t^{t}}{v^{2}}-\frac{x v}{4 b t}\right) d v
\end{aligned}
$$

This solution can best be expressed as a combination of complementary error functions in order to make use of the asymptotic properties of these wellknown functions. Hence

$$
\begin{aligned}
& G(x, p, t) \\
& \quad=\frac{1}{2}\left[\exp (-a c) \operatorname{erfc}\left\{\frac{1}{2} x t^{-1 / 2}-c t^{1 / 2}\right\}+\exp (a c) \operatorname{erfc}\left\{\frac{1}{2} x t^{-1 / 2}+c t^{1 / 2}\right\}\right],
\end{aligned}
$$


in which $\operatorname{erfc}(x)$ is the standard complementary error function with argument $x$ (see, [1, page 295]).

The closed form of the solution is obtained by inverting (3.9) using (3.3). Thus

$$
\begin{aligned}
& u(x, \varsigma, t)=\int_{0}^{\infty} \frac{F(p) H_{v}(p \varsigma) G(x, p, t)}{J_{v}^{2}\left(p \varsigma_{0}\right)+Y_{v}^{2}\left(p \varsigma_{0}\right)} p d p, \\
& c(x, \varsigma, t)=\varsigma^{-v} \exp \left(\frac{x}{2 b}\right) \int_{0}^{\infty} \frac{H_{v}(p \varsigma) F(p)}{J_{v}^{2}\left(p \varsigma_{0}\right)+Y_{v}^{2}\left(p \varsigma_{0}\right)} G(x, p, t) p d p .
\end{aligned}
$$

The function $F(p)$ must be found while its inverse is known, but the use of its inverse means the use of the convolution theorem for the Weber transform. In general, this leads to a rather complicated double integral. However, the resulting integral can be reduced to an integral in one variable for certain forms of the function $f(z)$. We will illustrate this by two examples discussed in Sections 4 and 5.

4. Uniform point source. When the source at $x=0$ is concentrated at a point $z=h$ (i.e., $f(z)=\delta(z-h)$ ) above ground level, the expression $F(p)$ reduces to

$$
F(p)=h_{0}^{v+1}, \quad h_{0}=2 h^{1 / 2}
$$

The problem posed here is relevant to industrial pollution where a chimney emits a pollutant into the surrounding environment.

The expressions (3.12) and (3.13) here reduce, respectively, to

$$
\begin{aligned}
& u(x, \varsigma, t)=\int_{0}^{\infty} \frac{H_{v}(p \varsigma) H_{v}\left(p h_{0}\right)}{J_{v}^{2}\left(p \varsigma_{0}\right)+Y_{v}^{2}\left(p \varsigma_{0}\right)} G(x, t ; p) p d p \\
& c(x, \varsigma, t)=\left(h_{0}\right)^{v+1} \varsigma^{-v} \exp \left(\frac{x}{2 b}\right) \int_{0}^{\infty} \frac{H_{v}(p \varsigma) H_{v}\left(p h_{0}\right)}{J_{v}^{2}\left(p \varsigma_{0}\right)+Y_{v}^{2}\left(p \varsigma_{0}\right)} G(x, t ; p) p d p
\end{aligned}
$$

The relatively simple solution (4.3) can be used to examine a number of limiting cases which may clarify the manner in which time variations influence the distribution of dust.

SPECiAl Limiting CASES. Here, we examine the general solution (3.13) in special cases where the solution is simpler and reduces to situations studied previously.

(i) Solution for large times. One of the purposes of this study is to examine the solution as $t \rightarrow \infty$ to see how it relates to the solution obtained for the steady-state problem in which $\partial / \partial t$ was set equal to zero from the outset [6, (3.12)]. This case should lead to the steady state that must ultimately develop 
for very large times. Now, (3.11) gives

$$
G(x, p, t) \approx e^{-a c} \operatorname{erfc}\left(-c t^{1 / 2}\right)+e^{a c} \operatorname{erfc}\left(c t^{1 / 2}\right) \text { for } t \rightarrow \infty .
$$

If we use the reflection property of the error function

$$
\operatorname{erfc}(-z)=2-\operatorname{erfc}(z)
$$

we obtain

$$
f(x, t ; p) \approx 2 e^{-a c}+2 \sinh (a c) \operatorname{erfc}\left(c t^{1 / 2}\right) \approx 2 e^{-a c} \text { for } t \rightarrow \infty
$$

and the solution (4.3) reduces to

$$
\begin{aligned}
& c(x, \varsigma, t) \\
& \quad=h_{0}^{v+1} \varsigma^{-v} \exp \left(\frac{x}{2 b}\right) \int_{0}^{\infty} \frac{H_{v}(p \varsigma) H_{v}\left(p h_{0}\right)}{J_{v}^{2}\left(p \varsigma_{0}\right)+Y_{v}^{2}\left(p \varsigma_{o}\right)} \exp \left(-\frac{x}{2 b} \sqrt{1+4 b p^{2}}\right) p d p
\end{aligned}
$$

which is identical to [6, (3.12)] for the steady-state solution.

(ii) $t \rightarrow 0$. When $t$ is very small, we have

$$
\begin{aligned}
G(x, p, t) & \approx e^{-a c} \operatorname{erfc}\left(\frac{1}{2} a t^{-1 / 2}\right)+e^{a c} \operatorname{erfc}\left(\frac{1}{2} a t^{-1 / 2}\right) \\
& \approx 2 \cosh (a c) \operatorname{erfc}\left(c t^{-1 / 2}\right) .
\end{aligned}
$$

Here, we appeal to the asymptotic property of the error function

$$
\begin{gathered}
\operatorname{erfc}(z) \approx(\pi z)^{-1 / 2} \exp \left(-z^{2}\right)\left\{1+\sum_{n=1}^{\infty}(-1)^{n} \frac{1 \cdot 3 \cdots(2 n-1)}{\left(2 z^{2}\right)^{n}}\right\}, \quad|z| \rightarrow \infty, \\
|\arg z| \leq \frac{\pi}{2}-\delta, \quad \delta>0,
\end{gathered}
$$

to find that $c(x, \varsigma, t)$ becomes zero as $t$ vanishes.

(iii) $b \rightarrow 0$. This is the situation in which horizontal diffusion is absent. Since the argument of the first error function in (3.11) can take positive and negative values, we must consider the cases (iiia) $a / 2 t-c t>0$ and (iiib) $a / 2 t-c t<0$ separately. For (iiia), the utilization of the asymptotic form (4.9) of the error 


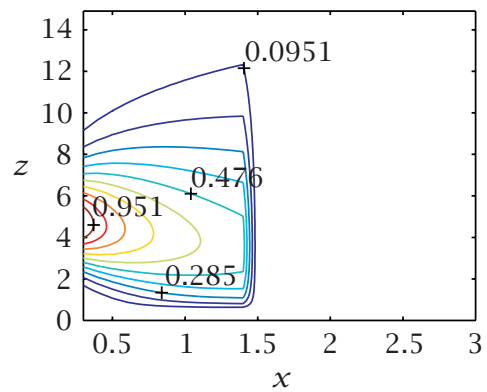

(a)

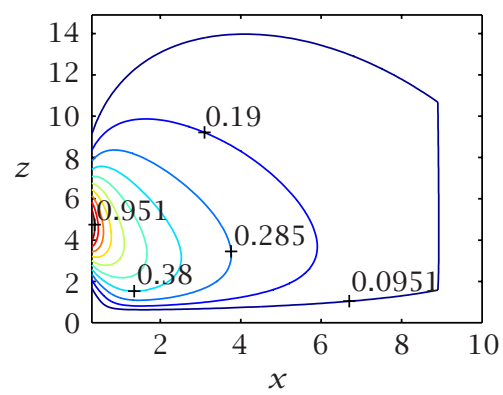

(c)

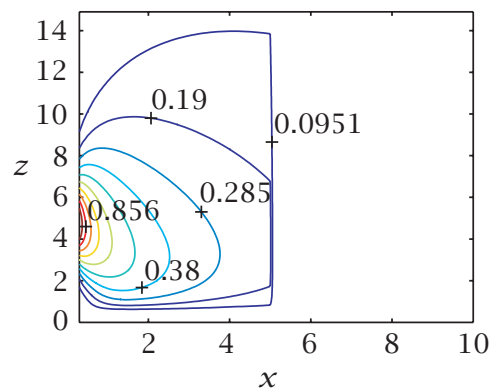

(b)

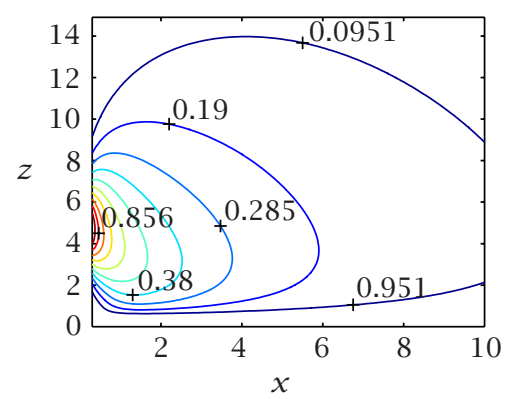

(d)

FIGURE 4.1. The isolines of the concentration of the point source in the $(x, z)$ plane when $h=5.0, v=0.5$, and $z_{0}=0.5$ for (a) $t=1.5$, (b) $t=5.0$, (c) $t=9.0$, and (d) $t=50.0$, in the case when there is no horizontal diffusion.

function leads to

$$
G(x, p, t) \approx \frac{x \sqrt{b t}}{\sqrt{2 \pi}} \exp \left\{-\frac{x^{2}+t^{2}}{4 b t}\right\} \rightarrow 0 \quad \text { as } b \longrightarrow 0
$$

but in case (iiib), we use both properties (4.5) and (4.9) of the error function to find that

$$
f(x, t ; p) \approx \exp \left[-x p^{2}-\frac{x}{2 b}\right]+\frac{x \sqrt{b t}}{\sqrt{2 \pi}} \exp \left\{-\frac{x^{2}+t^{2}}{4 b t}\right\} \quad \text { as } b \longrightarrow 0 .
$$

The solution in the case $b=0$ then has the form

$$
c(x, \varsigma, t)=h_{0}^{v+1} \varsigma^{-v} H(t-x) \int_{0}^{\infty} \frac{H_{v}(p \varsigma) H_{v}\left(p h_{0}\right)}{J_{v}^{2}\left(p \varsigma_{0}\right)+Y_{v}^{2}\left(p \varsigma_{0}\right)} e^{-x p^{2}} p d p
$$

which can be obtained by integrating the equations directly for $b=0$ (see Figure 4.1). 
5. Vertical source of finite length. Consider a source situated along $x=0$ such that (see (2.10c))

$$
f(z)= \begin{cases}1 & \text { for } z_{0}<z<L \\ 0 & \text { for } z>L,\end{cases}
$$

so that the pollutant is emitted uniformly throughout the distance between the heights $z=z_{o}$ and $z=L$ along the vertical line $x=0$. This situation is of great relevance to the spread of dust in arid and semiarid lands where desertification is a major problem (see, e.g., [3]).

The solution is obtained by using the expression (5.1) into (3.7), (3.9), and (3.12). We find that

$$
F(p)=Y_{v}\left(p \varsigma_{0}\right) I_{1}-J_{v}\left(p \varsigma_{0}\right) I_{2}
$$

in which

$$
I_{1}=\int_{\varsigma_{0}}^{L} \varsigma^{v+1} Y_{v}(p \varsigma) d \varsigma, \quad I_{2}=\int_{\varsigma_{0}}^{L} \varsigma^{v+1} J_{v}(p \varsigma) d \varsigma
$$

For $\varsigma_{0} \rightarrow 0$, these two integrals can be evaluated analytically (see [8, page 683]). They reduce to

$$
I_{1}=\frac{\ell^{v+1} J_{v+1}(p \ell)}{p}, \quad I_{2}=\frac{\ell^{v+1} Y_{v+1}(p \ell)}{p}+\frac{2^{v+1} \Gamma(v+1)}{\pi p^{v+2}},
$$

in which $\Gamma(x)$ is the Gamma function of argument $x$. It then follows that

$$
p F(p)=\frac{\ell^{v+1}}{p}\left[Y_{v}(p \varsigma) J_{v+1}(p \ell)-J_{v}(p \varsigma) Y_{v+1}(p \ell)\right]-\frac{2^{v+1}}{\pi p^{v+2}} \Gamma(\nu+1) .
$$

The integral (3.13) together with (5.5) has been computed for various values of the parameters $v, L$, and $b$ in the $(x, \varsigma, t)$ space and a sample of the results is given in Figures 6.5 and 6.6.

6. Discussion. The expression (3.13) for the concentration $c(x, \varsigma, t)$ was integrated numerically for the two cases of a point source (discussed in Section 4) and of a vertical uniform source (discussed in Section 5), for various values of the parameters. The results of the point source are presented in Figures 4.1, $6.1,6.2,6.3$, and 6.4 while those of the vertical source are illustrated in Figures 6.5 and 6.6. The steady state of the point source problem has already been studied [6] and we here concentrated on it with a view to examining the influence of the time factor and the development of the steady state from the initial state assumed here. The results for the example of the vertical source have been limited to the investigation of the evolution of the solution with time as well as to the illustration of the different behaviour due to the different initial conditions. 


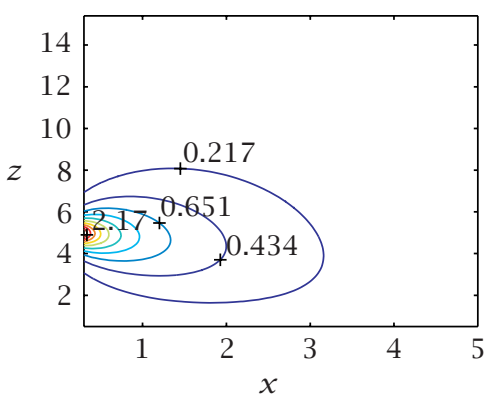

(a)

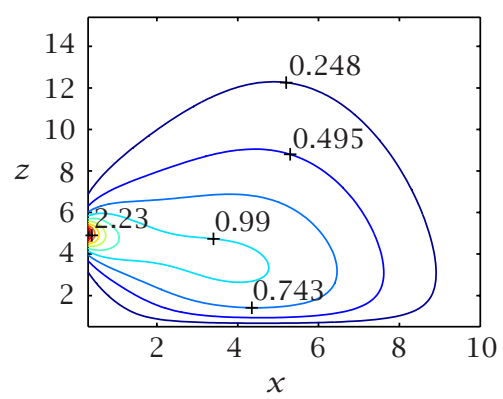

(c)

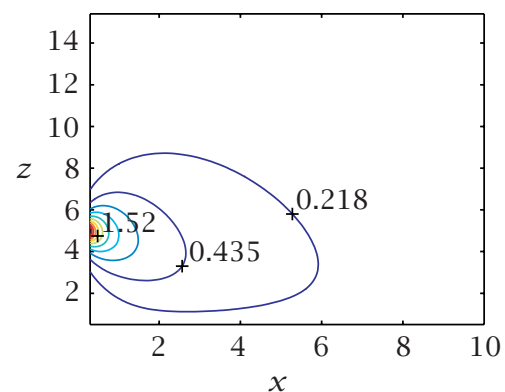

(b)

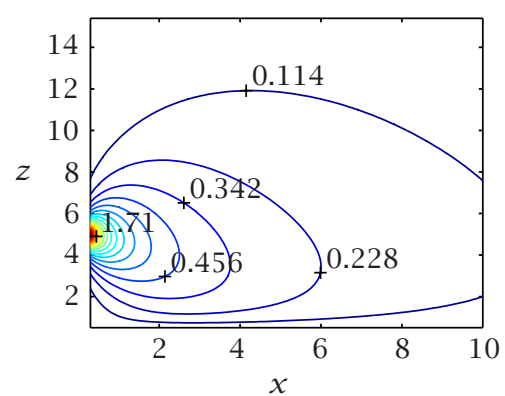

(d)

FIGURE 6.1. The isolines of the concentration of the point source in the $(x, z)$ plane when $h=5.0, v=0.5, z_{0}=0.5$, and $b=1.0$ for (a) $t=2.0$, (b) $t=5.0$, (c) $t=9.0$, and (d) $t=50.0$. Compare with Figure 4.1 and note the influence of the horizontal diffusion.

In Figures 4.1 and 6.1, the contours of the concentration in the $(x, z)$ plane are drawn for different values of the time for two different values of the parameter $b$ representing the component of horizontal diffusion. It is observed that for small and moderate values of the time, the two distributions differ in two aspects. When $b=0$ and horizontal diffusion is negligible, the concentration of dust advances in the $x$-direction with a vertical front advancing at the uniform horizontal speed of the basic flow, while for nonzero values of $b$, the diffusion in the horizontal direction permits the advancement of dust particles ahead of the vertical line advancing at the uniform speed of the flow. The other aspect in which the two cases differ concerns the spread of the concentration in the vertical direction. As the horizontal component of diffusion increases, the spread of the dust in the vertical direction is reduced in the sense that the height to which it can diffuse is reduced by the increase in the horizontal component of diffusion. When time increases indefinitely, the distribution of dust looks similar for both $b=0$ and $b \neq 0$. 


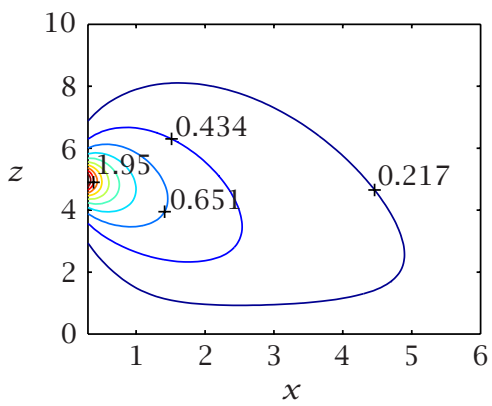

(a)

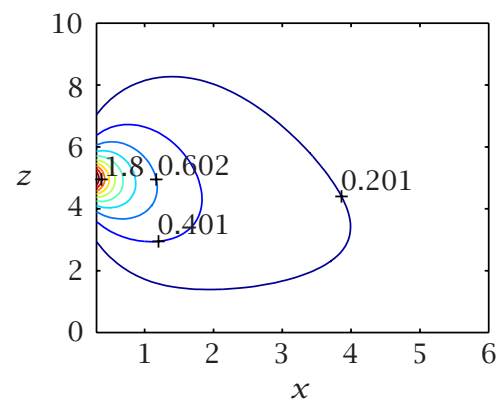

(c)

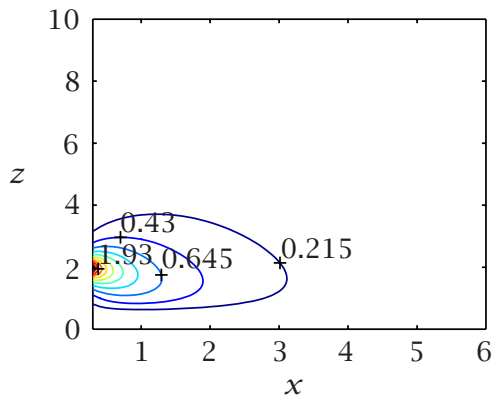

(b)

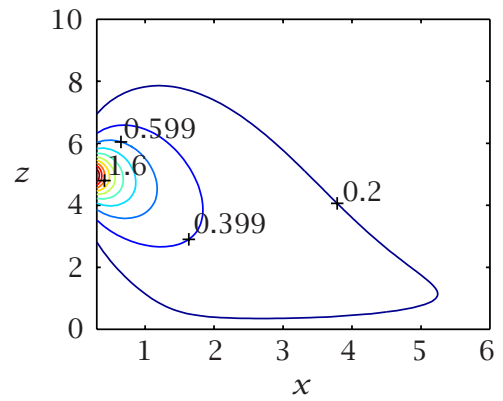

(d)

FIGURE 6.2. The isolines of the concentration of the point source for different sets of values of parameters illustrating the effects of the parameters $h, v, b$, and $z_{0}$ for a fixed value of time, $t=5.0$. (a) $h=5.0, v=1.0, z_{0}=0.5, b=1.0$; (b) $h=2.0, v=1.0, z_{0}=0.5$, $b=1.0$; (c) $h=5.0, v=1.0, z_{0}=0.5, b=3.0$; (d) $h=5.0, v=1.0$, $z_{0}=0.1, b=1.0$.

Whereas the parameter representing horizontal diffusion provides a strong influence on the evolution of the dust with time, the remaining parameters play a more subservient role. The influence of the parameters $h, v$, and $z_{o}$ is illustrated in Figure 6.2. Comparing Figures 6.1(b) and 6.2(a), we see that the increase in $v$ (representing an increase in the settling velocity and hence stronger gravitational effect) limits the spread of the dust above the source and tends to force it to settle to the ground. The increase in the height of the source enhances the spread of the dust in the horizontal direction since it allows the particles to acquire relatively higher velocities before they reach the ground, as can be seen by comparing Figures 6.2(a) and 6.2(b). The roughness height $z_{o}$ does not seem to have a strong influence on the evolution of the dust (see Figures 6.2(a) and 6.2(d)). 


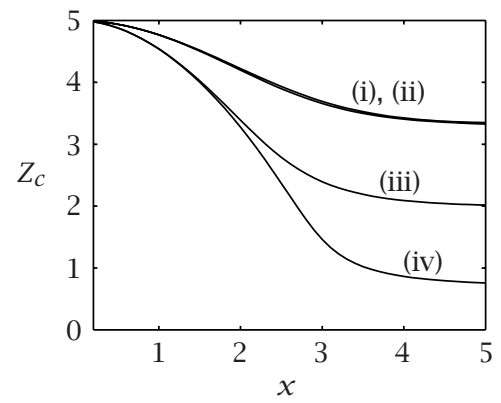

(a)

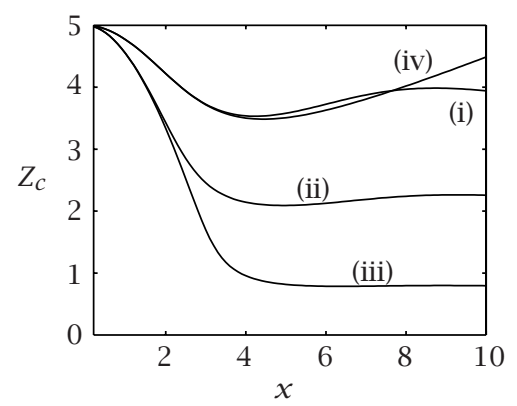

(c)

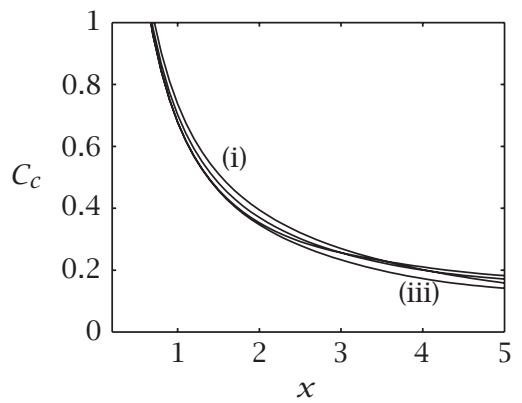

(b)

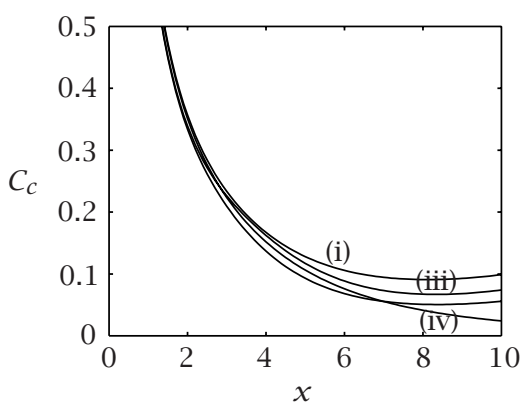

(d)

FiguRE 6.3. An illustration of the dependence of the curve of extensive pollution, height $Z_{c}$, and concentration $C_{c}$ along it on the parameters of the point source problem. (a) and (b) correspond to $t=5.0, h=5.0$, and (i) $z_{0}=0.5, v=0.5, b=2.0$; (ii) $z_{0}=0.5, v=0.5$, $b=3.0$; (iii) $z_{o}=0.5, v=1.5, b=2.0$; and (iv) $z_{0}=0.1, v=0.5$, $b=2.0$, respectively. (c) and (d) again correspond to $h=5.0$, and (i) $z_{0}=0.5, v=0.5, b=3.0, t=10.0$; (ii) $z_{0}=0.5, v=1.5, b=3.0$, $t=10.0$; (iii) $z_{0}=0.1, v=1.5, b=3.0, t=10.0$; and (iv) $z_{0}=0.5$, $v=0.5, b=3.0, t=50.0$, respectively.

For all values of the parameter $b$ in the case of a point source, the concentration of dust possesses a curve of extensive pollution. This is defined as the curve on which, for every value of $x$, the concentration is maximized over $z$. The profile of this curve, which was identified in the steady-state solution [6], is here found to evolve with time, in addition to its dependence on the parameters of the problem. This is illustrated in Figures 6.3 and 6.4. Figure 6.3 illustrates the dependence of the profile of the curve on the parameters at moderate times. It is noticeable that the value of the concentration of dust along the curve does not vary very much with the parameters, but the height varies significantly, particularly, with variations in $v$ and $z_{0}$. Figure 6.4 illustrates the evolution of the curve with time for different sets of values of the parameters. 


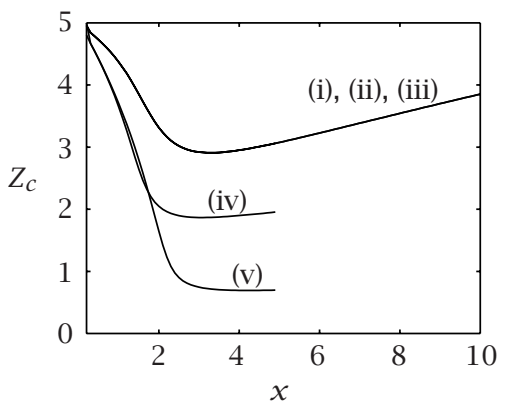

(a)

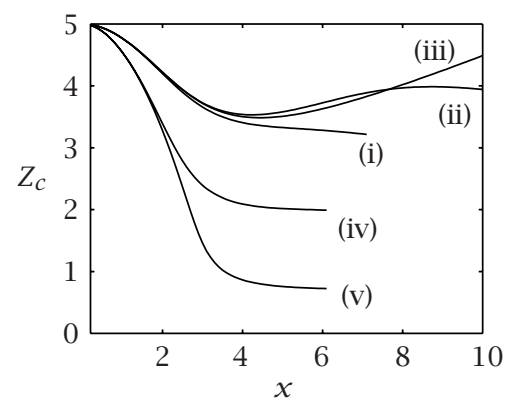

(c)

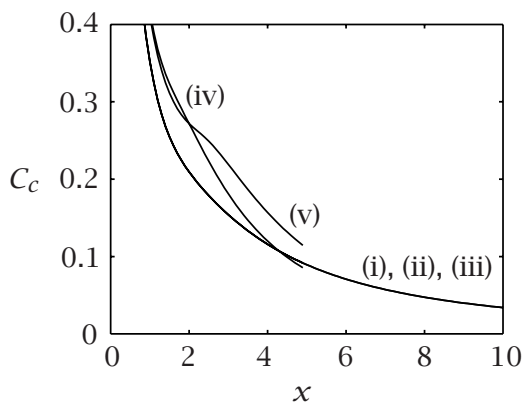

(b)

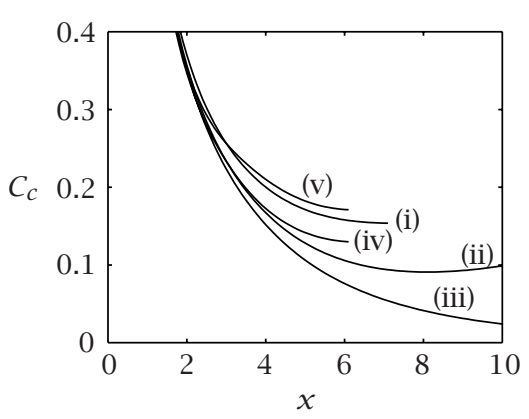

(d)

FIGURE 6.4. A sample of the data obtained for the curve of extensive pollution of dust in the $(x, z)$ plane for different values of time and two values of $b$ when the source is situated at one point; (a), (b) for $b=0$ and (c), (d) for $b=3.0$. Figures 6.4(a) and 6.4(c) represent the variations in height of the point with horizontal distance $x$ while (b) and (d) represent the profiles of the concentration as a function of $x$ when $h=5.0$. The curves refer to the sets of data: (i) $t=5.0$, $v=0.5, z_{0}=0.5$, (ii) $t=10.0, v=0.5, z_{o}=0.5$, (iii) $t=50.0, v=0.5$, $z_{0}=0.5$, (iv) $t=5.0, v=1.5, z_{0}=0.5$, and (v) $t=5.0, v=0.5$, $z_{0}=0.1$.

Since the evolution of the dust as it spreads into the $(x, z)$ plane with the increase of time depends crucially on the horizontal component of diffusion, we have illustrated such development of the curve of extensive pollution for two different values of $b$. Figures 6.4(a) and 6.4(c) give the profile of the curve in the $(x, z)$ plane for $b=0,3.0$, respectively, while (b) and (d) give the values of the concentration along the curve in the two corresponding cases. It is immediately clear from comparing Figures 6.4(a) and 6.4(c) that the height of the curve does not change very much with time, as shown by the curves (i), (ii), and (iii), although it shows small changes with time when the horizontal component of diffusion is nonzero. More dramatic changes are brought about by changes 


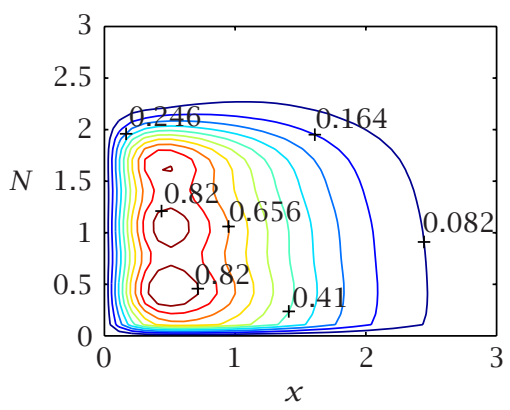

(a)

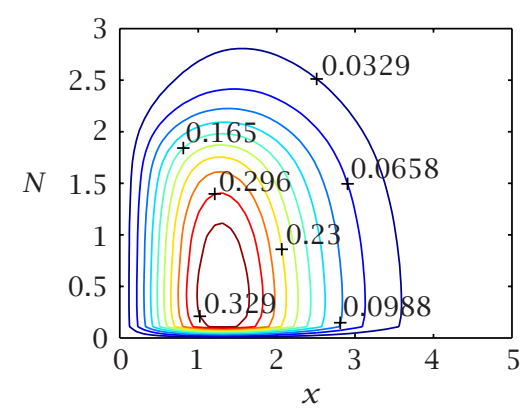

(c)

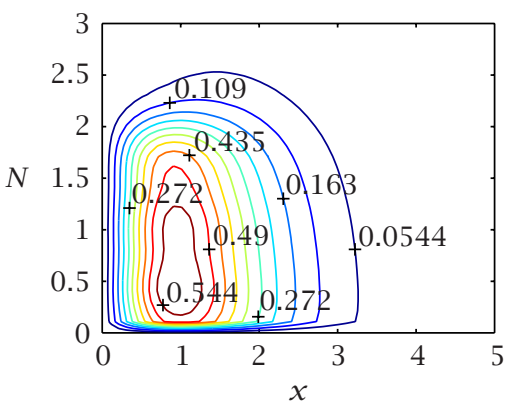

(b)

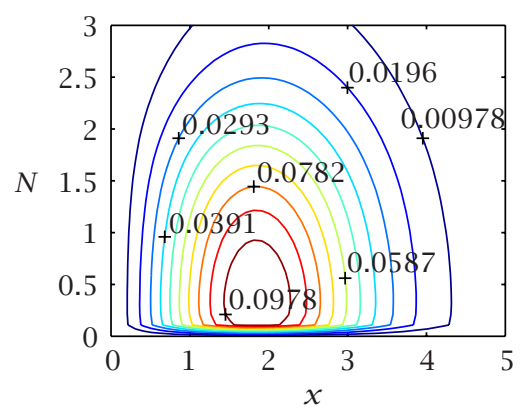

(d)

FIGURE 6.5. A sample of the results for the computation of the expression for the concentration of dust in the case of a finite vertical source when $v=0.5, b=0.5$, and $L=1.0$ for different times: (a) $t=1.0$, (b) $t=5.0$, (c) $t=10.0$, and (d) $t=20.0$. Note the slow movement of the point of accumulation with increasing time.

in $v$ and $z_{o}$. The behaviour of the concentration on the curve is different. In the absence of horizontal diffusion, the concentration does not change with time (Figure 6.4(b), (i), (ii), (iii)). When horizontal diffusion is present, the curve is displaced downwards as it evolves with time (Figure 6.4(d), (i), (ii), (iii)). The changes due to the different parameters $v$ and $z_{o}$ are small, as can be observed in Figures 6.4(b) and 6.4(d).

The contours of the concentration of dust with time in the case of the vertical uniform source are illustrated in Figure 6.5 for different values of the scaled time, $t$, for fixed $v, b$, and $L$. It is evident that a point of accumulation of dust, where the concentration is maximum, develops in the $(x, z)$ plane behind the advancing front of dust particles. The location of the point of accumulation depends on time but it is clear that it advances slowly with time and its height decreases gradually as time increases. Figure 6.6 illustrates the influence of the parameters on the distribution of dust at a fixed instant of time. Comparison of 


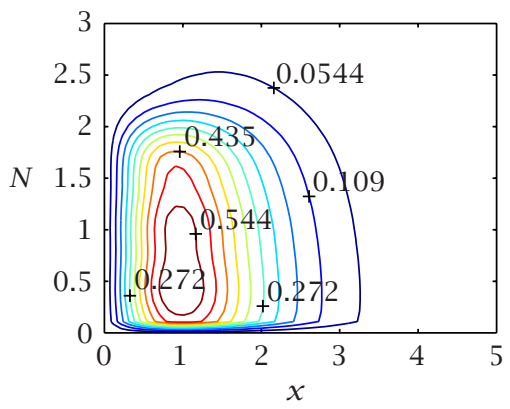

(a)

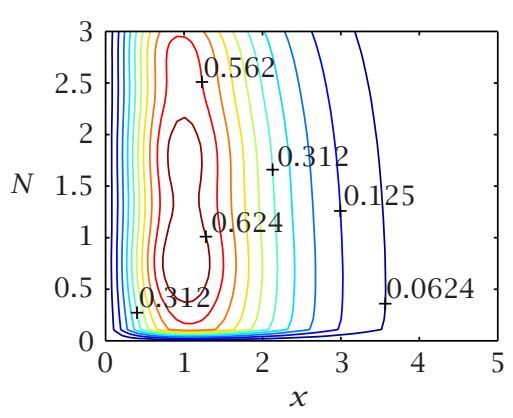

(c)

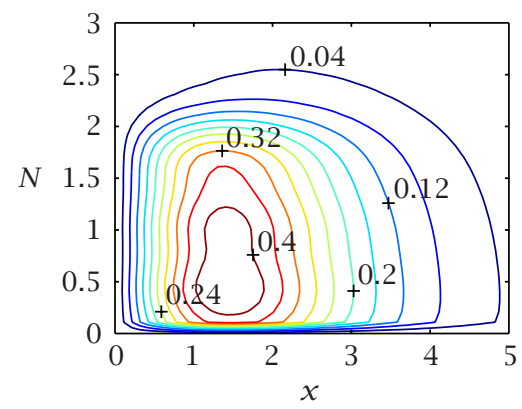

(b)

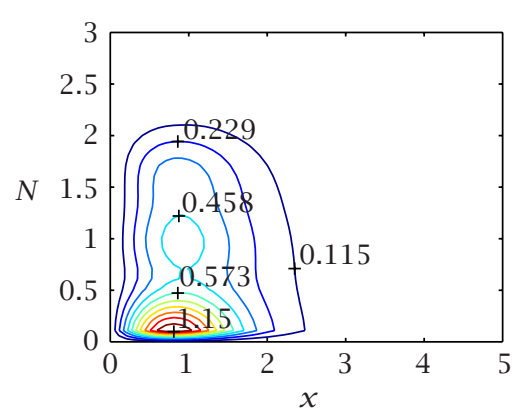

(d)

FiguRE 6.6. The contours of the concentration of dust for the vertical source at a fixed time, $t=5.0$, for different sets of the parameters: (a) $v=0.5, b=0.5, L=1.0$, (b) $v=0.5, b=1.5, L=1.0$, (c) $v=0.5, b=0.5, L=3.0$, and (d) $v=2.0, b=0.5, L=1.0$.

Figures 6.6(a) and 6.6(b) shows that the increase in horizontal diffusion (i.e., in $b$ ) promotes the spread of dust and enhances the advance of the accumulation point. The increase in the extent of the source (i.e., in $L$ ) naturally promotes the spread of dust vertically while the increase in the settling velocity (i.e., in $v$ ) forces the dust to settle down to the ground, as can be seen in Figure 6.6(d).

7. Conclusion. The initial value problem posed by fixed uniform sources of pollutant above ground level has been investigated. Adopting a uniform wind profile in the presence of both vertical and horizontal diffusions, the solution is obtained in closed form for all time. The behaviour of the evolution of the dust as it spreads in the $(x, z)$ vertical plane containing the source is examined in detail to illustrate its dependence on the parameters involved. These are the roughness height, $z_{0}$, the settling velocity, as represented by $v$, and the relative importance of the horizontal diffusion as compared to the vertical component of diffusion, as represented by the parameter $b$. It is found 
that the most significant change in the solution is due to the presence of the horizontal component of diffusion. In its absence, the dust spreads into space in such a way that its front is vertical and has the uniform horizontal speed of the wind while it diffuses vertically. The presence of the horizontal component of diffusion destroys the sharp vertical nature of the advancing front and the dust diffuses ahead of the vertical line moving horizontally with the speed of the flow. Whether the horizontal diffusion is strong or weak, the distribution of dust possesses a curve of extensive pollution which evolves in a manner dependent on the parameters of the problem but its height is weakly dependent on time as it extends in space with increasing time.

The behaviour of the solution for large times has been examined in detail with a view to assessing the manner in which a steady state is achieved. It was found that in the case of a point source, as time increases indefinitely, the solution uniformly approaches the steady-state solution obtained previously [6] by solving the equations in the absence of time variation.

The problem posed by a uniform vertical finite source was also discussed to illustrate the strong dependence of the solution on the type of source under consideration. It is found that the general influence of the parameters representing the components of diffusion and settling velocity is very similar but the distribution of the dust in the $(x, z)$ plane is drastically different.

\section{REFERENCES}

[1] M. Abramowitz and I. A. Stegun (eds.), Handbook of Mathematical Functions, with Formulas, Graphs, and Mathematical Tables, National Bureau of Standards, Applied Mathematics Series, vol. 55, U.S. Government Printing Office, District of Columbia, 1965.

[2] J. G. Bartzis, Turbulent diffusion modeling for wind flow and dispersion analysis, Atmospheric Environment 23 (1989), 1963-1969.

[3] H. E. Dregne, Desertification of arid lands, Physics of Desertification (F. El-Baz and M. H. A. Hassan, eds.), Martinus Nijhoff Publishers, Dordrecht, 1986, pp. 4-34.

[4] F. El-Baz, I. A. Eltayeb, and M. H. A. Hassan (eds.), Sand Transport and Desertification in Arid and Semi-Arid Lands, World Scientific Publishers, Singapore, 1990.

[5] I. A. Eltayeb and M. H. A. Hassan, On the non-linear evolution of sand dunes, Geophys. J. R. Astr. Soc. 65 (1981), 31-45.

[6] _ Diffusion of dust particles from a point source above ground level and a line source at ground level, Geophys. J. Internat. 142 (2000), 426-438.

[7] D. A. Gillette, Threshold friction velocities for dust production for agricultural soils, J. Geophys. Res. 93 (1988), 12645-12662.

[8] I. S. Gradshteyn and I. M. Ryzhik, Table of Integrals, Series, and Products, Academic Press, New York, 1980.

[9] M. H. A. Hassan and I. A. Eltayeb, Suspension of transport of wind eroded sand particles, Geophys. J. Internat. 104 (1991), 147-152.

[10] J. F. Kennedy, The formation of sediment ripples, dunes and anti-dunes, Ann. Rev. Fluid Mech. 1 (1969), 147-168. 
[11] J. S. Lin and L. Hildemann, A generalized mathematical scheme to analytically solve the atmospheric diffusion equation with dry deposition, Atmospheric Environment 31 (1997), 59-71.

[12] R. P. Llwelyn, An analytical model for transport, dispersion and elimination of air pollutants emitted from a point source, Atmospheric Environment 17 (1983), 249-256.

[13] O. F. T. Roberts, The theoretical scattering of smoke in a turbulent atmosphere, Proc. Roy. Soc. London Ser. A 104 (1923), 640-654.

[14] R. A. Schmidt, Vertical profiles of wind speed, snow concentration, and humidity in blowing snow, Boundary-Layer Meteorol. 23 (1982), 223-246.

[15] M. Sharan, M. P. Singh, and A. K. Yadav, Mathematical model for atmospheric dispersion in low winds with eddy diffusivities as linear functions of downwind distance, Atmospheric Environment 30 (1996), 1137-1145.

[16] E. L. Skidmore, Soil erosion by wind: an overview, Physics of Desertification (F. ElBaz and M. H. A. Hassan, eds.), Martinus Nijhoff Publishers, Dordrecht, 1986, pp. 261-273.

[17] M. Takeuchi, Vertical profile and horizontal increase of drift snow transport, J. Glaciol. 26 (1980), no. 94, 481-492.

I. A. Eltayeb: Department of Mathematics and Statistics, College of Science, Sultan Qaboos University, P.O. Box 36, Al-Khodh 123, Muscat, Sultanate of Oman

E-mail address: ie1tayeb@squ. edu.om

M. H. A. Hassan: Third World Academy of Sciences, International Centre for Theoretical Physics, P.O. Box 586, Miramare 11, 34100 Trieste, Italy

E-mail address: hassan@ictp.trieste.it 


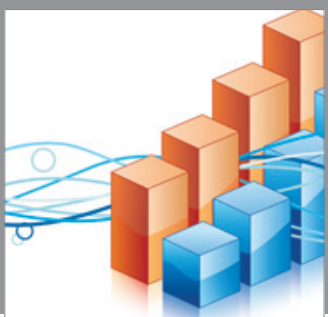

Advances in

Operations Research

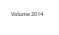

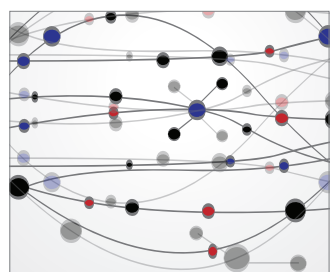

\section{The Scientific} World Journal
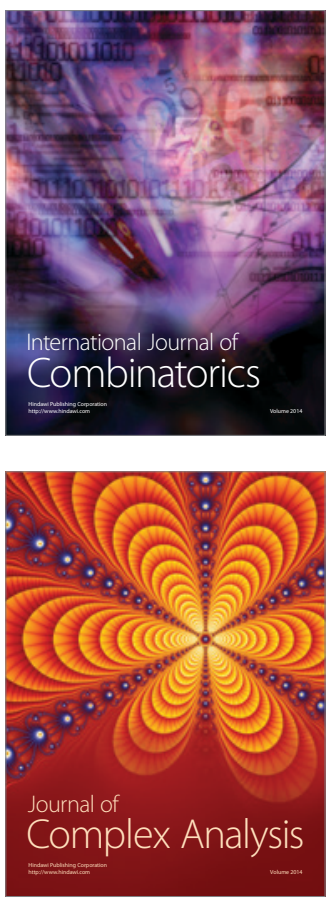

International Journal of

Mathematics and

Mathematical

Sciences
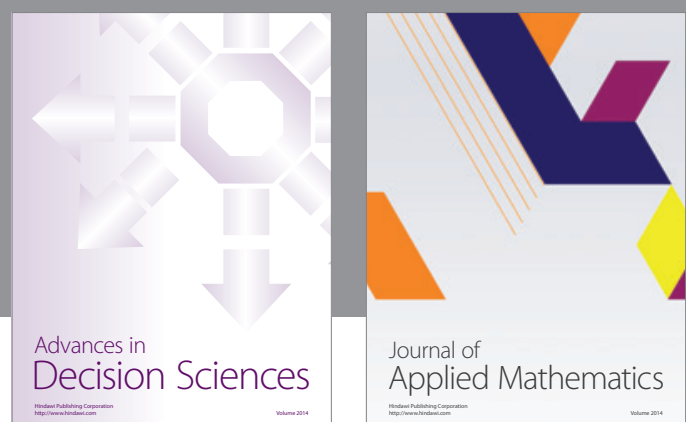

Journal of

Applied Mathematics
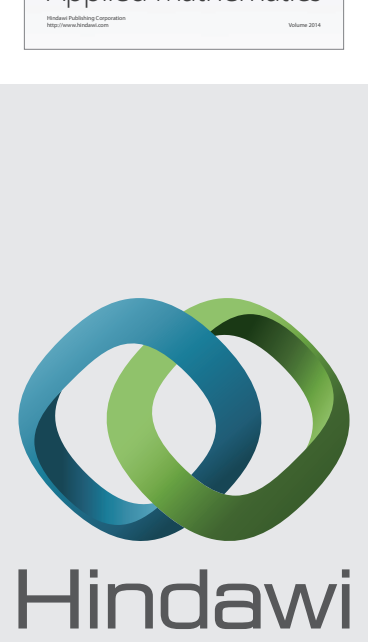

Submit your manuscripts at http://www.hindawi.com
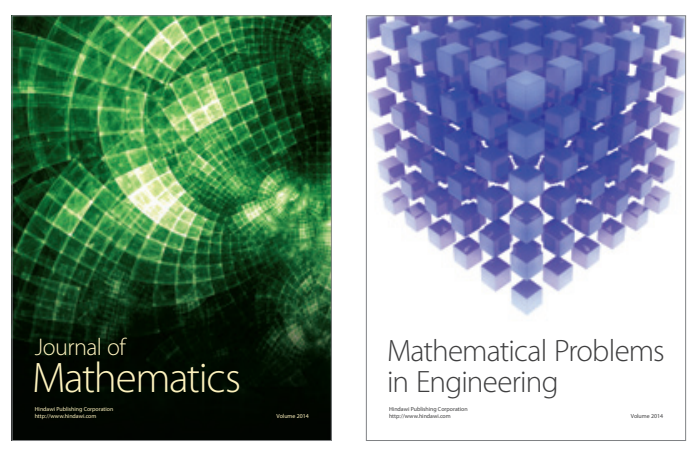

Mathematical Problems in Engineering
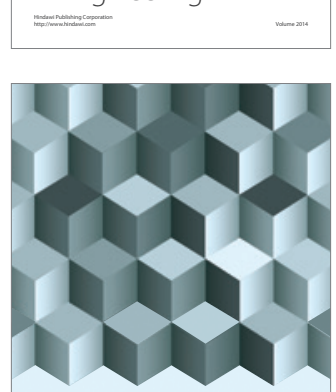

Journal of

Function Spaces
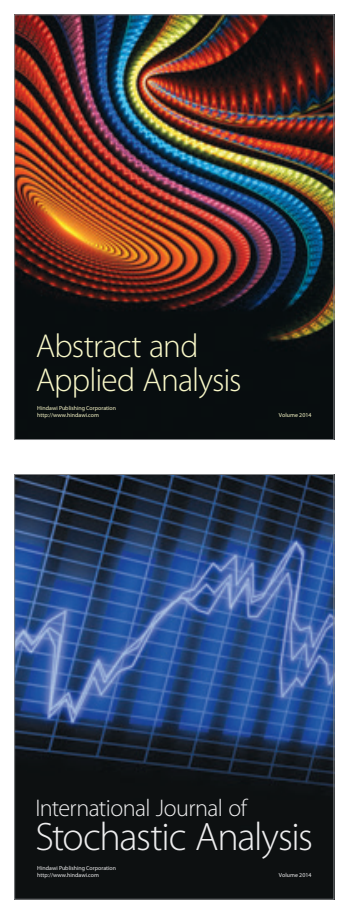

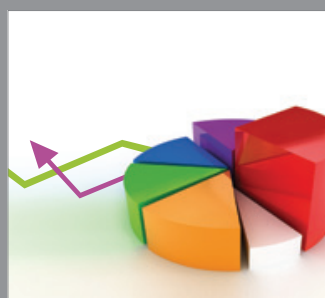

ournal of

Probability and Statistics

Promensencen
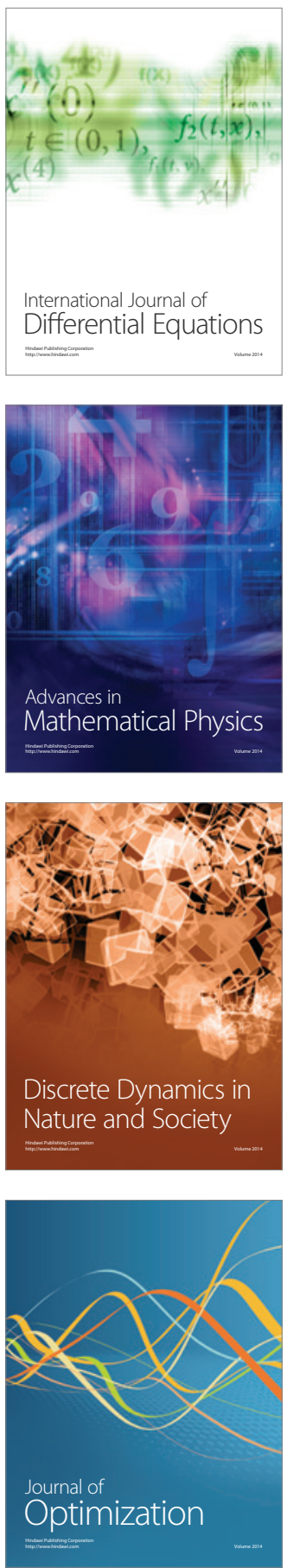